AperTO - Archivio Istituzionale Open Access dell'Università di Torino

\title{
Dual-frequency irradiation CEST-MRI of endogenous bulk mobile proteins
}

\section{This is a pre print version of the following article:}

Original Citation:

Availability:

This version is available http://hdl.handle.net/2318/1716617

since 2019-11-20T16:23:27Z

Published version:

DOI:10.1002/nbm.3920

Terms of use:

Open Access

Anyone can freely access the full text of works made available as "Open Access". Works made available under a Creative Commons license can be used according to the terms and conditions of said license. Use of all other works requires consent of the right holder (author or publisher) if not exempted from copyright protection by the applicable law. 


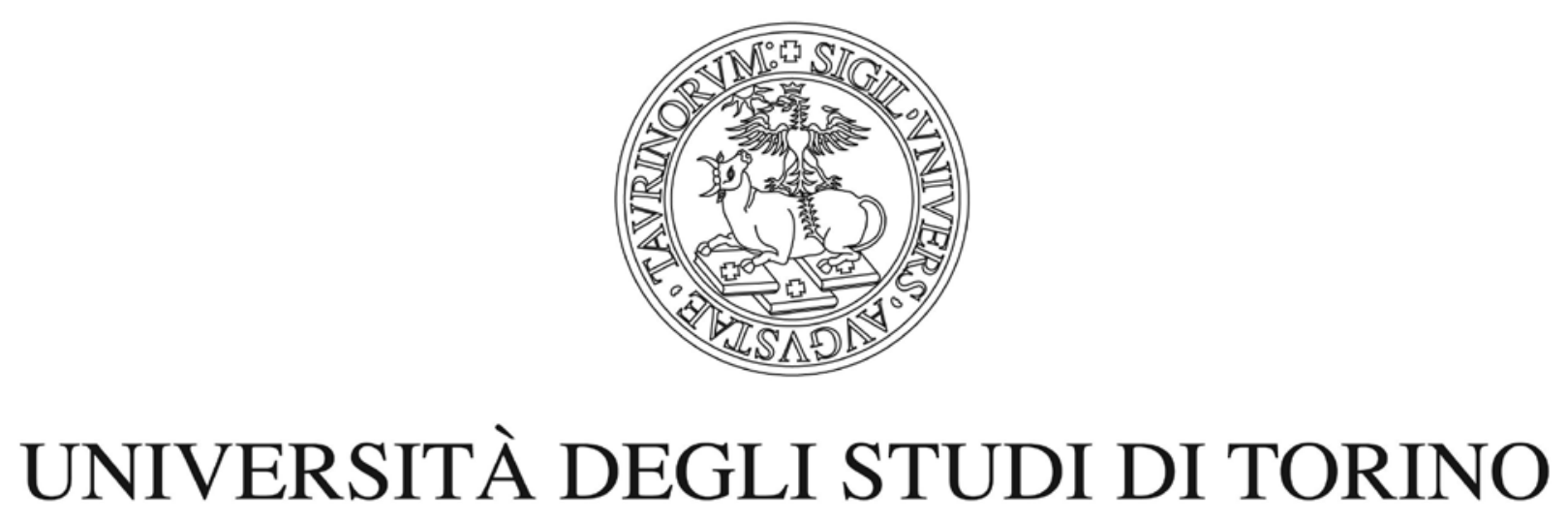

This is an author version of the contribution published on:

Steffen Goerke, Johannes Breitling, Moritz Zaiss, Johannes Windschuh, Patrick Schuenke, Daniel Paech, Dario Livio Longo, Karel D. Klika, Mark E. Ladd, and Peter Bachert

Dual-frequency irradiation CEST-MRI of endogenous bulkmobile proteins

In NMR in Biomedicine

The definitive version is available at:

DOI: $10.1002 / \mathrm{nbm} .3920$ 


\title{
In vivo protein imaging \\ by means of dual frequency irradiation chemical exchange saturation transfer (dualCEST)
}

\begin{abstract}
Steffen Goerke ${ }^{1 * \dagger}$, Johannes Breitling ${ }^{1 *}$, Moritz Zaiss ${ }^{1,2}$, Johannes Windschuh ${ }^{1}$, Patrick Schuenke ${ }^{1}$, Daniel Paech ${ }^{3,4}$, Dario Livio Longo ${ }^{5}$, Karel D. Klika ${ }^{6}$, Mark E. Ladd ${ }^{1}$, and Peter Bachert $^{1}$
\end{abstract}

1 Division of Medical Physics in Radiology

3 Neurooncologic Imaging, Department of Radiology

6 Molecular Structure Analysis

Deutsches Krebsforschungszentrum [German Cancer Research Center] (DKFZ)

Im Neuenheimer Feld 280

69120 Heidelberg

Baden-Württemberg

Germany

2 Max-Planck-Institute for Biological Cybernetics

Department of High-field Magnetic Resonance

Spemannstraße 41

72076 Tübingen

Baden-Württemberg

Germany

4 Department of Neuroradiology

University of Heidelberg Medical Center

Im Neuenheimer Feld 672

69120 Heidelberg

Baden-Württemberg

Germany

5 Istituto di Biostrutture e Bioimmagini (IBB)

Consiglio Nazionale delle Ricerche (CNR)

Via Nizza 52

10126 Torino

Italy

* These authors contributed equally to this work

† Corresponding author:

Dr. rer. nat. Steffen Görke

Im Neuenheimer Feld 280

69120 Heidelberg

Baden-Württemberg

Germany

Phone: +49-6221/42-2543

E-mail: s.goerke@dkfz.de

The authors declare no conflict of interest.

\section{Word Count}

Main text: 3514 (max. 5000) / Methods: 768 (max. 3000) 


\section{Abbreviations}

$\begin{array}{ll}\Delta \omega & \text { frequency offset } \\ \Delta \omega_{c} & \text { constant frequency offset } \\ \text { AREX } & \text { apparent exchange-dependent relaxation } \\ B_{0} & \text { static magnetic field strength } \\ B_{1} & \text { mean RF amplitude } \\ \text { BSA } & \text { bovine serum albumin } \\ \text { CEST } & \text { chemical exchange saturation transfer } \\ \text { CSF } & \text { cerebrospinal fluid } \\ \text { DC } & \text { duty cycle } \\ \text { dualCEST } & \text { dual frequency irradiation CEST } \\ \text { FL } & \text { fluorescence spectroscopy } \\ \text { L } & \text { Lorentzian-shaped function } \\ M_{0} & \text { equilibrium water magnetization } \\ M_{\text {sat }} & \text { water magnetization after pre-saturation } \\ \text { MRI } & \text { magnetic resonance imaging } \\ \text { PBS } & \text { phosphate-buffered saline } \\ R_{1 w} & \text { longitudinal relaxation rate of water } \\ R F & \text { radio frequency } \\ \text { rNOE } & \text { relayed nuclear Overhauser effect } \\ \text { SDS } & \text { sodium dodecyl sulfate } \\ \text { SNR } & \text { signal-to-noise ratio } \\ \text { sSMT } & \text { semi-solid magnetization transfer } \\ T_{1 w} & \text { longitudinal relaxation time of water } \\ T_{2 w} & \text { transversal relaxation time of water } \\ T_{\Delta \omega c}(\Delta \omega) & \text { saturation crosstalk } \\ t_{p} & \text { pulse length } \\ t_{d} & \text { interpulse delay } \\ T_{\text {protein }} & \text { isolated saturation crosstalk of proteins } \\ T_{\text {ssMT }} & \text { isolated saturation crosstalk of semi solid macromolecular structures } \\ Z & \text { Z-spectrum } \\ Z_{\text {ref }} & \text { reference Z-spectrum }\end{array}$




\begin{abstract}
We developed a novel magnetic resonance imaging (MRI) contrast allowing the unbiased detection of proteins in vivo. Proteins are important macromolecules involved in nearly every biological process of living organisms. Alterations in protein expression are associated with several types of disease, including e.g. cancer or Alzheimer's. An imaging methodology selective to such pathological modifications is therefore of particular interest. Assignment of the presented approach - dual frequency irradiation chemical exchange saturation transfer (dualCEST) - to proteins was verified experimentally in the presence of other cellular compounds. The unique specificity is based on a newly introduced dimension of selectivity: measuring the coupling of different CEST signals among each other. Moreover, this allowed us to overcome limitations resulting from comparatively low static magnetic field strengths, and thus, to include dualCEST examinations into daily routine diagnostic protocols at clinically approved MR tomographs. Image data of a tumor patient, allowed the identification of infiltrated brain regions demonstrating the value of dualCEST as a diagnostic tool.
\end{abstract}

\title{
Keywords
}

MRI, CEST, magnetization transfer, proteins, cancer 


\section{Introduction}

Proteins are important macromolecules that are crucial for nearly every biological process of living organisms. Depending on the structure they function as e.g. (i) transporters for small molecules and ions, (ii) stabilizer of the cell structure, (iii) regulator of growth and cellular differentiation, or (iv) catalysts of biochemical reactions ${ }^{1}$. Malfunction or overexpression of specific proteins can be either a symptom or also the cause of various diseases, making proteins a unique biomarker for cellular stress, aging and disease ${ }^{2}$. Especially for the diagnosis of cancer, proteins are of particular interest, as cancer comes along with profound changes in gene expression, i.e. uncontrolled biosynthesis of proteins ${ }^{3,4}$. Moreover, neurodegenerative diseases like e.g. Alzheimer's can be identified by the pathological deposition of aggregated proteins $^{5,6}$.

Non-invasive imaging of bulk proteins can be achieved by chemical exchange saturation transfer (CEST) $)^{7-11}$ magnetic resonance imaging (MRI) and was already successfully applied to diagnostic imaging ${ }^{12-15}$. CEST image data for example allowed to assess tumor malignancy ${ }^{16}$, to differentiate radiation necrosis ${ }^{17,18}$ or to identify ischemic stroke ${ }^{19,20}$. CEST is a spectroscopic imaging technique that exploits the spontaneous chemical exchange of protons between solutes and water in order to generate a signal amplification. This is realized by a frequency selective pre-saturation of chemically exchanging protons in solutes and a subsequent MRI of the modified water signal. Therefore, CEST enables imaging of proteins with nearly the sensitivity of conventional water proton MRI. However, in living tissue also CEST signals of other cellular compounds such as e.g. metabolites ${ }^{21-25}$, lipids ${ }^{14,26}$, or semisolid macromolecular structures ${ }^{27-29}$ can be obtained, which are interfering with the signals of proteins (Fig. 1). Moreover, CEST signals depend on various physiological parameters that determine the chemical exchange processes e.g. $\mathrm{pH}$ or temperature $\mathrm{e}^{9,30-32}$. On the one hand, this multi parametric dependency makes CEST a promising imaging technique with the potential to depict a wide range of physiological processes, but on the other hand, the numerous parameters are at the same time a large drawback in terms of specificity. Even though, particular adjustments of the CEST pulse scheme (i.e. tuning of the pre-saturation amplitude $B_{1}$ or the interpulse delay $t_{d}$ ) allow to design exchange rate filters ${ }^{33-}$ ${ }^{35}$ that highlight the signal component of proteins, so far, these signals remain to be diluted by other cellular compounds. Consequently, the actual contribution of proteins to CEST signal changes in different pathologies remains to be clarified.

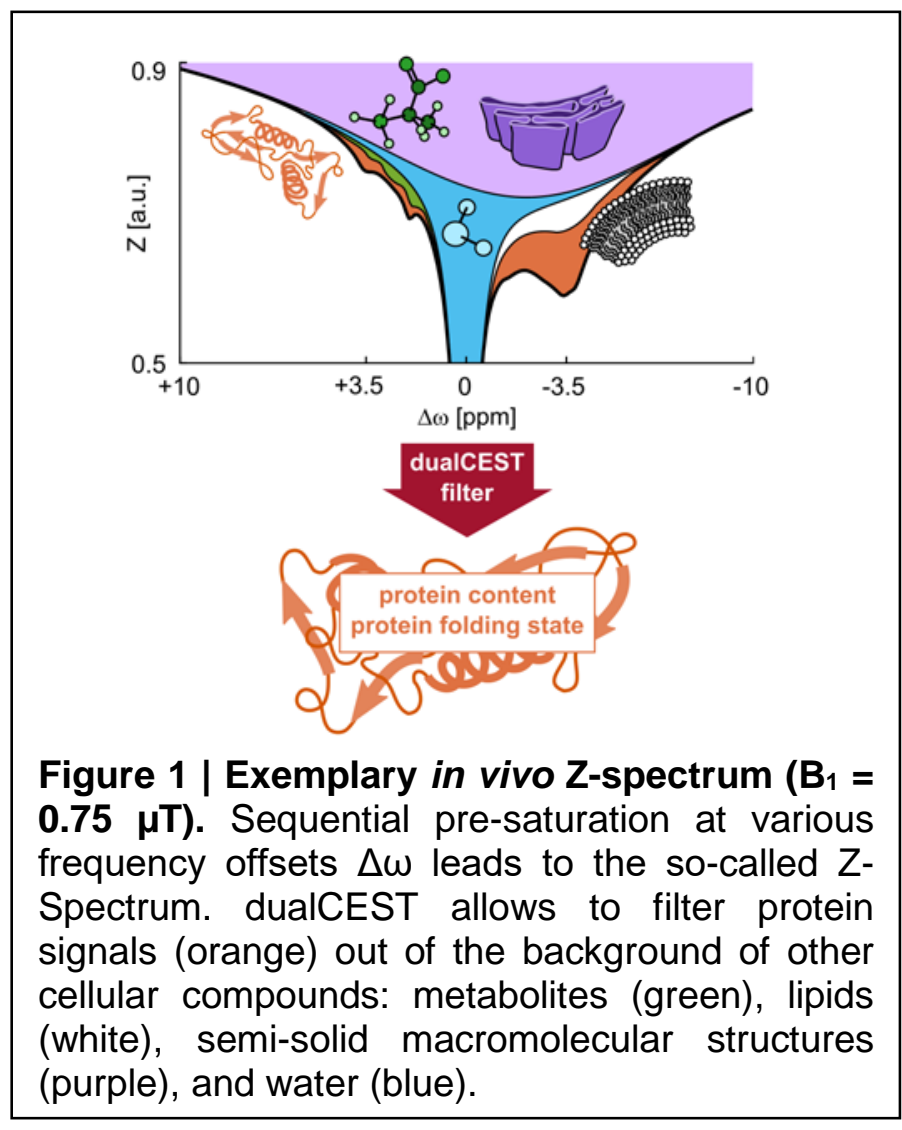

Here we present a novel MRI methodology - dual frequency irradiation CEST (dualCEST) that allows for the first time the unbiased detection of proteins in vivo. The dualCEST signal solely depends on (i) protein content (i.e. concentration and proteome composition) as well as (ii) on the protein folding state, which allows imaging of aberrant structural and conformational changes of proteins ${ }^{36-39}$. Specificity of the dualCEST signal is achieved by the introduction of a novel dimension of selectivity: dualCEST depicts the cross magnetization transfer between 
CEST signals resonating at two different frequency offsets. Remarkably, this eliminates the need for ultra-high static magnetic field strengths $\mathrm{B}_{0} \geq 7 \mathrm{~T}$ that are required for adequate separation of individual resonances using conventional CEST-MRI. In addition, the presented approach profits from lower magnetic field strengths allowing inclusion of dualCEST examinations into daily routine diagnostic protocols at clinically approved MR tomographs $\left(\mathrm{B}_{0}\right.$ $\leq 3 \mathrm{~T}$ ). Assignment of the dualCEST signal to proteins is demonstrated by the successive addition of different cellular compounds to a protein model solution. The potential of protein dualCEST-MRI for diagnostic imaging was investigated in a multimodal study of a brain tumor patient. Hence, dualCEST enables the unbiased detection of proteins at clinical field strength which allows to identify pathological modifications of the proteome in vivo. 


\section{Results}

The dualCEST approach. To demonstrate the idea of the dualCEST approach a protein solution containing bovine serum albumin (BSA) is investigated. dualCEST detects the MR signal of water after an alternating radio frequency (RF) irradiation at two different frequency offsets $\Delta \omega$ and $\Delta \omega_{c}$ (Fig. 2a). This allows simultaneous sampling of the conventional Z-spectrum as a function of $\Delta \omega$ while constantly saturating CEST signals at $\Delta \omega_{c}$ (Fig. 2b). For a detailed analysis of the influence of the constant saturation, the CEST signals of proteins are separated from the direct water saturation $(\Delta \omega=0 \mathrm{ppm})$ by the apparent exchange-dependent relaxation $\left(\right.$ AREX) ${ }^{40}$ evaluation (Fig. 2c). Constant saturation at $\Delta \omega_{c}=-3.0 \mathrm{ppm}$ leads to a significant reduction of protein CEST signals on the other side of the water resonance $(\Delta \omega>0 \mathrm{ppm})$. Hence, protein CEST signals in the positive and negative frequency region are to some extent coupled via a magnetization transfer pathway. The amount of magnetization transfer between two particular frequency offsets - in the following termed as saturation crosstalk $\mathrm{T}_{\Delta \omega c}(\Delta \omega)$ - can be quantified by subtraction of the AREX spectra. Quantification by simple subtraction of the two spectra is justified due to the linearity of CEST signals using the AREX evaluation. Remarkably, in proteins the saturation crosstalk is in the order of $30-40 \%$ of the conventional AREX spectrum demonstrating the potential for its application in vivo. The observed saturation crosstalk is

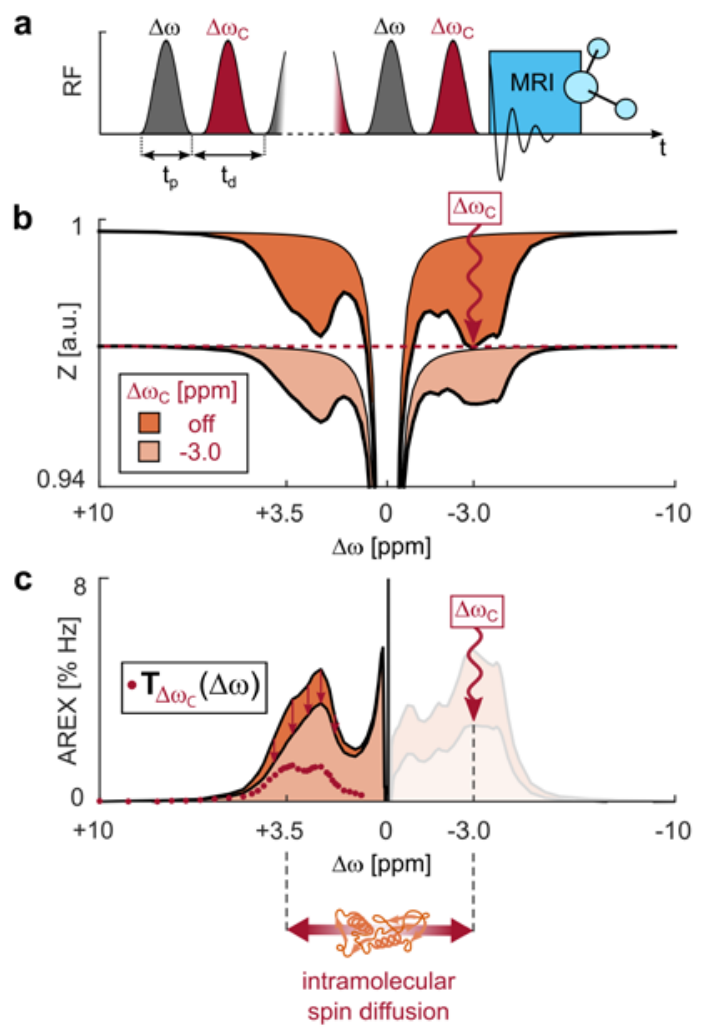

Figure 2 | The dualCEST approach. (a) Scheme of the dualCEST pulse sequence. (b) Z-spectra of BSA proteins $\left(B_{1}=0.45 \mu \mathrm{T}\right)$ with and without constant saturation at $\Delta \omega_{c}$. (c) Isolated CEST signals using the AREX evaluation. The saturation crosstalk $T_{\Delta \omega c}(\Delta \omega)$ (red dots) is defined by the difference between the two spectra.

attributed to intra-molecular spin diffusion processes ${ }^{41}$ between dipolar-coupled protons. Spin diffusion is also known to mediate the intra-molecular magnetization transfer of relayed nuclear Overhauser effect (rNOE) CEST signals, which were studied extensively in the past few years ${ }^{26,42,14,35}$.

Rearrangement of the mathematical expression of $\mathrm{T}$ allows calculation of the saturation crosstalk without the need for prior AREX evaluation (Supporting Information, Fig. S1):

$$
\mathrm{T}_{\Delta \omega_{\mathrm{C}}}(\Delta \omega)=\frac{\mathrm{R}_{1 \mathrm{w}}}{\mathrm{DC}} \cdot\left[\frac{1}{\mathrm{Z}_{\text {off }}(\Delta \omega)}+\frac{1}{\mathrm{Z}_{\Delta \omega_{C}}(\mathrm{off})}-\frac{1}{\mathrm{Z}_{\Delta \omega_{C}}(\Delta \omega)}-\frac{1}{\mathrm{Z}_{\text {off }}(\text { off })}\right]
$$

with the longitudinal relaxation rate of water $R_{1 w}$ and the duty cycle of the saturation pulse scheme DC. This has the advantage that $\mathrm{T}_{\Delta \omega c}(\Delta \omega)$ can be calculated without the need for estimation of the direct water saturation by a fitting procedure. Consequently, the saturation crosstalk between two particular frequency offsets is characterized by only four Z-values (equation (1)) allowing fast and direct acquisition which is crucial for in vivo application. 
The advantage of the dualCEST signal in comparison to the conventional CEST approach is that it provides a novel dimension of selectivity. As dualCEST depicts the intra-molecular magnetization transfer between two different CEST signals, selectivity to compounds that exhibit more than one resonance in the Z-spectrum can be achieved. Consequently, by setting the two frequency offsets to the positive and negative range $\left(\Delta \omega>0\right.$ and $\left.\Delta \omega_{c}<0 \mathrm{ppm}\right)$ the dualCEST signal is selective to proteins, because only proteins offer individual resonances on both sides of the Z-spectrum (Fig. 1). However, as the comparatively broad resonance of the semi-solid magnetization transfer (SSMT) ranges also from positive to negative frequency offsets, an additional contribution to the dualCEST signal of macromolecular structures can be expected. To retrieve the isolated signal of proteins, a correction method has to be applied. 
Correction for signals of semi-solid macromolecular structures. To investigate the dualCEST signal of proteins in the presence of semi-solid macromolecular structures, an ex vivo tissue homogenate derived from pig brain is prepared. Indeed, the dualCEST spectrum acquired with similar sequence parameters as in figure $2\left(\Delta \omega_{c}=-3.5 \mathrm{ppm}\right)$ exhibits a comparatively broad saturation crosstalk with a spectral width larger than $10 \mathrm{ppm}$ (Fig. 3b, red line). Consequently, the acquired signal comprises, in addition to proteins, a component originating from semi-solid macromolecular structures. To isolate the superimposed signal of proteins $T_{\text {protein }}$ the exceptional selectivity of the dualCEST approach is utilized (Fig. 3a): by shifting the constant saturation offresonant from protein signals while remaining on-resonant to the ssMT $\left(\Delta \omega_{c}=-10 \mathrm{ppm}\right)$ the spectral profile of the underlying SSMT component can be sampled in an isolated manner (Fig. 3b, magenta line). The actual amplitude of the signal $T_{\text {ssm }}$ (Fig. 3b, black line) is determined by scaling the spectral profile by the factor $\mathrm{T}_{-3.5}(+10) / \mathrm{T}_{-10}(+10)$ (Fig. 3b, gray dots). This calculus does not simply pose an estimation but rather can be derived in an analytical way (Supporting Information, Fig. S2). In conclusion, the isolated signal of proteins in the presence of semi-solid macromolecular structures can be determined by:

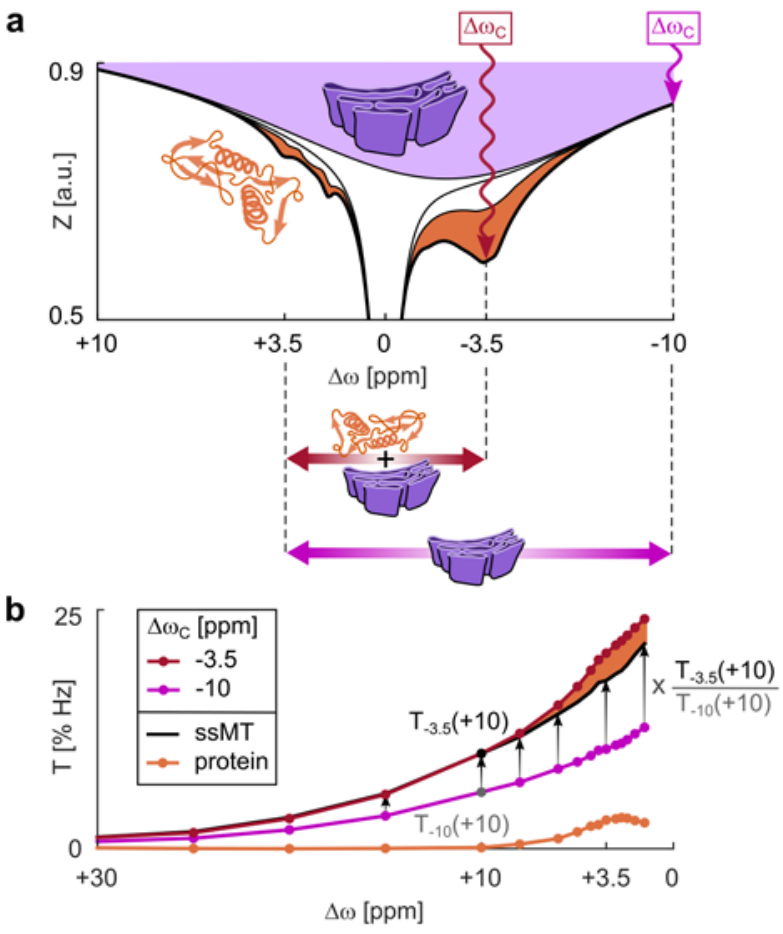

Figure 3 | ssMT correction. (a) Z-spectrum of ex vivo brain tissue homogenate $\left(B_{1}=0.75 \mu \mathrm{T}\right)$. By selection of $\Delta \omega_{c}$ selectivity of the dualCEST signal to different cellular compounds can be achieved. (b) Scaling of the spectral profile of signals originating from semi-solid macromolecular structures $\mathrm{T}_{-10}(\Delta \omega)$ (purple line) allows to retrieve the unbiased signal of proteins (orange line).

$$
T_{\text {protein }}(\Delta \omega)=T_{-3.5}(\Delta \omega)-\underbrace{\frac{T_{-3.5}(+10)}{T_{-10}(+10)} \cdot T_{-10}(\Delta \omega)}_{T_{\text {sSMT }}}
$$

This enables application of the dualCEST approach to investigate alterations of the proteome in living organisms. For calculation of $T_{\text {protein }}$ in total nine $Z$-values are required leading to approximately only a doubling in acquisition time compared to the uncorrected signal (equation (1)). In addition, still no fitting procedure is required allowing fast determination of $T_{\text {protein }}$ in vivo without the need for sampling of a whole spectrum at various $\Delta \omega$. The consistency of the spectral widths of $T_{\text {protein }}$ in tissue homogenates (Fig. 3b, orange line) and $T$ in purified protein solutions (Fig. 2c) hints towards proper functioning of the presented SSMT correction. However, to unambiguously verify the assignment of the dualCEST signal to proteins, different cellular compounds are added successively to a protein model solution. 
Characterization of the protein dualCEST signal. As commonly known, signals of different cellular compounds are spectrally overlapped in Z-spectra in vivo (Fig. 1). To mimic this situation protein solutions are prepared containing (i) BSA, as well as different amounts of (ii) metabolites (i.e. carnosine and creatine) and (iii) lipids purified from mouse brain tissue (Fig. 4a). Besides the CEST signals of BSA in the positive and negative frequency region (Fig. 4b, orange line) individual resonances of carnosine, creatine and tissue-lipids are resolved around $\Delta \omega=+3.5,+2.0$ and -3.5 ppm, respectively (Fig. $4 \mathrm{~b}$ ). In addition, a broad SSMT of tissue-lipids can be obtained (Fig. 4a, blue lines) completing the imitated in vivo Zspectrum. Remarkably, although Zspectra strongly depend on the added cellular compounds, the dualCEST signal remains constant (Fig. 4c). This verifies the assignment of $T_{\text {protein }}$ to signals originating solely from proteins. Furthermore, $T_{\text {protein }}$ is compensated for changes in water signal relaxation (Fig. 4d) enabling unbiased investigation of the proteome in living organisms.

To identify parameters that in turn affect the amplitude of the dualCEST signal, protein solutions under different physiological conditions (i.e. concentration, folding state, and $\mathrm{pH}$ ) are investigated (Fig. 5). As expected, $T$ increases linearly as a function of protein content (Fig. 5a). Of note, at high concentrations $\mathrm{c}>10 \%(\mathrm{w} / \mathrm{v})$ an amplified increase is observed (Fig. 5b, dashed line) which can be explained by a progressive immobilization of large molecules in a crowded environment of high protein density. Besides its obvious dependence on concentration, also a considerable variation of $\mathrm{T}$ as a function of the protein folding state (i.e. molecular structure and conformation) is expected. The reason for this is the origin of the dualCEST signal from intra-molecular spin diffusion processes that, in turn, strongly depend on the interaction distances of the involved nuclei ${ }^{41}$. Following previous studies $^{37,38}$, unfolding of BSA is introduced by the detergent sodium dodecyl sulfate (SDS) and screened by
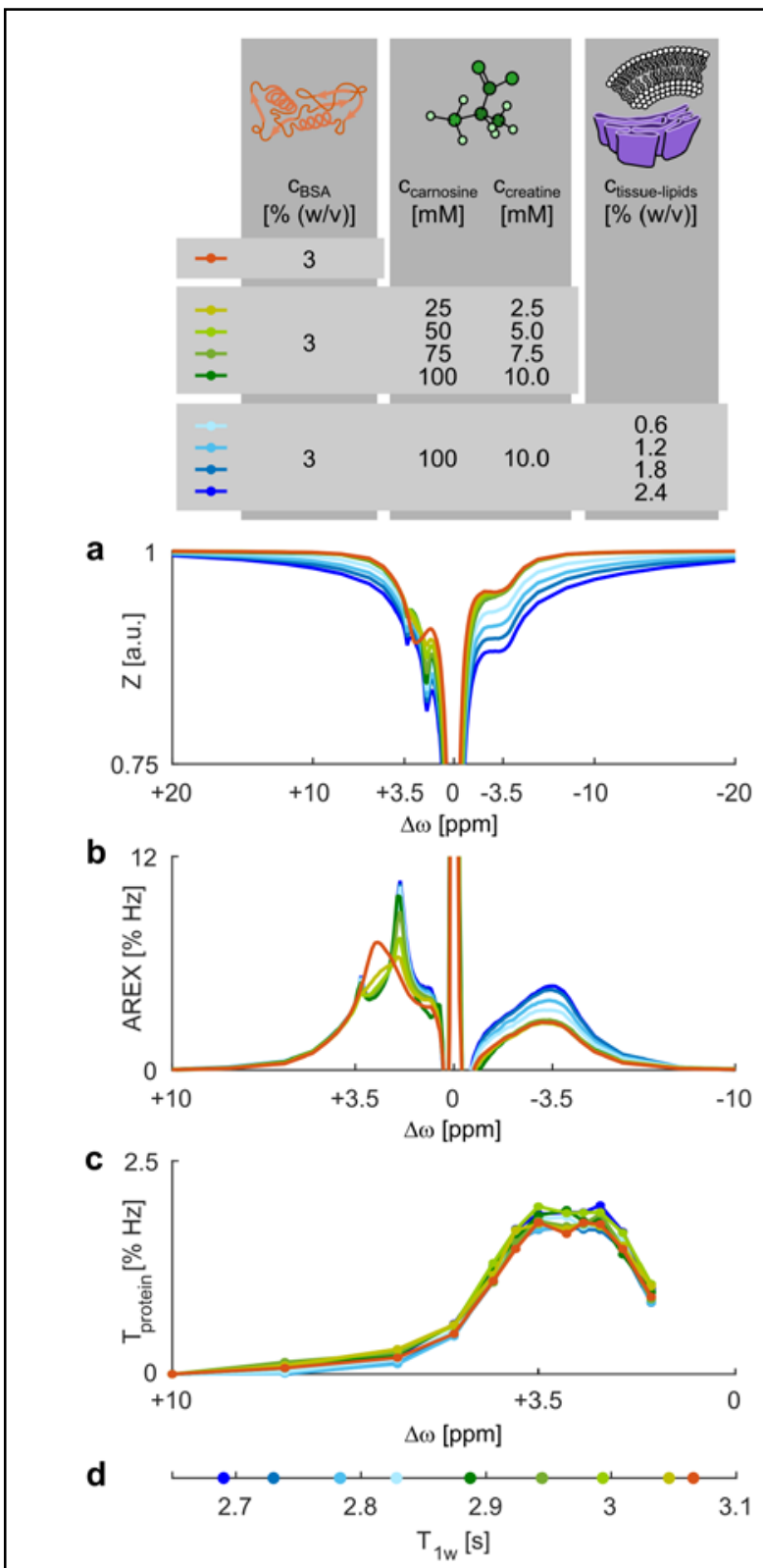

Figure 4 | Assignment of the dualCEST signal to proteins. (a) Z-, (b) AREX- and (c) T-spectra of BSA proteins for varying concentrations of cellular compounds $\left(\Delta \omega_{\mathrm{C}}=-3.5 \mathrm{ppm}, \mathrm{B}_{1}=1.5\right.$ $\mu \mathrm{T})$. (d) With an increasing amount of compounds the longitudinal relaxation time of water is reduced.

fluorescence spectroscopy (Fig. 5d, dashed line). Indeed, an exceptional good correlation of $\mathrm{T}$ with the protein folding state is observed. This verifies the potential of the dualCEST signal to image aberrant structural and conformational changes of proteins. Finally, the $\mathrm{pH}-$ dependence was investigated in order to demonstrate the robustness of the dualCEST signal against changes in the chemical exchange properties (i.e. exchange rate). As expected, $\mathrm{T}$ remains constant in the physiological range between $\mathrm{pH} 7$ and 7.4 (Fig. 5f, gray shaded). 
Significant signal changes in the lower $\mathrm{pH}$ range $<6.5$ most likely originate also from partial protein unfolding in an acid environment ${ }^{43}$.
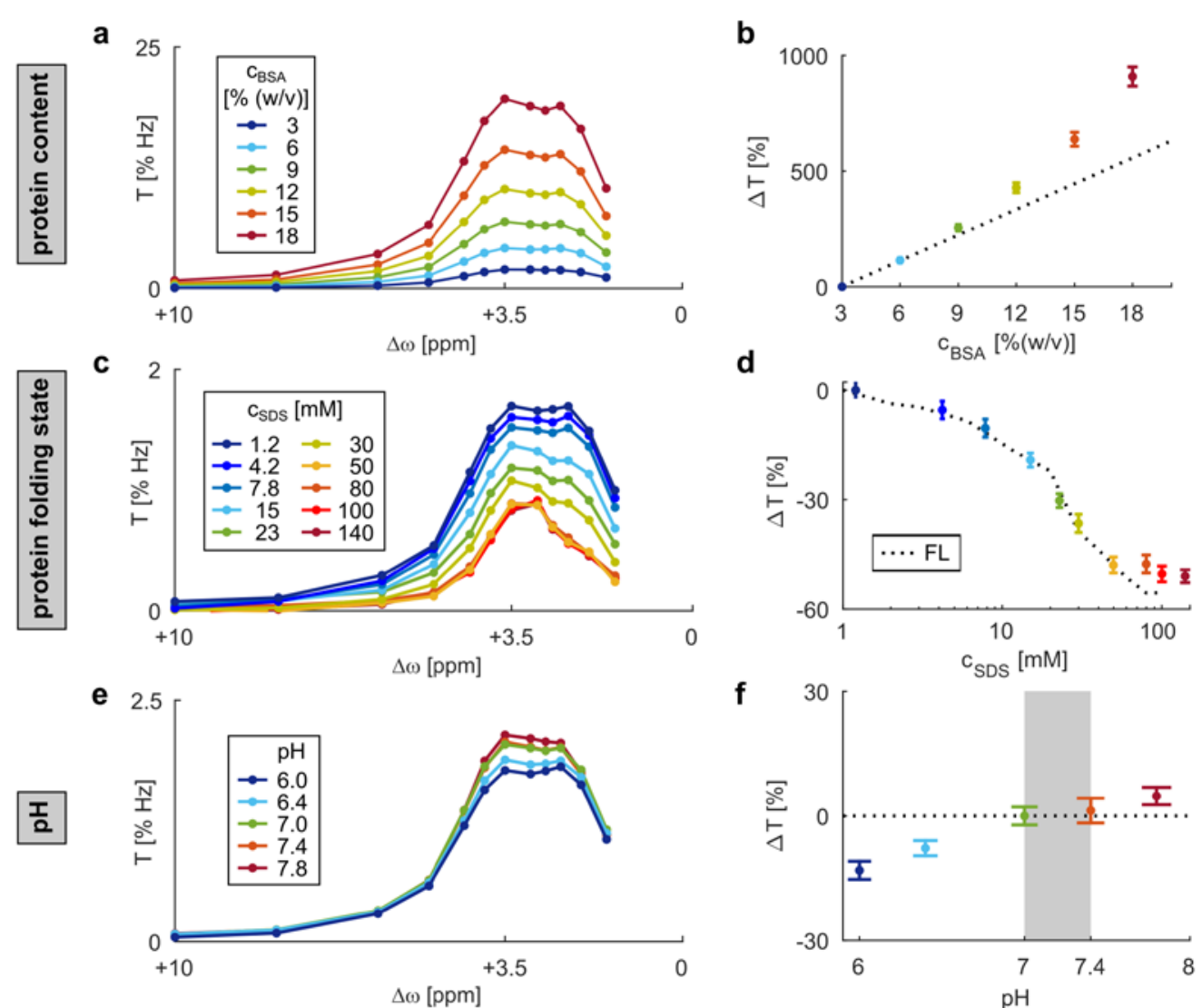

Figure 5 | Dependence of the dualCEST signal on physiological parameters. T-spectra of BSA proteins $\left(\Delta \omega_{c}=-3.5 \mathrm{ppm}, \mathrm{B}_{1}=1.5 \mu \mathrm{T}\right)$ for different (a) concentrations, (c) folding states, and $(\mathbf{e}) \mathrm{pH}$. $(\mathbf{b}, \mathbf{d}, \mathbf{f})$ Percentage variation of $\mathrm{T}$ at $\Delta \omega=+3.5 \mathrm{ppm}$. (d) As a reference, the protein folding state was determined by fluorescence spectroscopy (FL).

In summary, the dualCEST signal $T_{\text {protein }}$ presented in this study solely depends on (i) protein content and on the (ii) protein folding state. Contributions from other cellular compounds, water relaxation properties, or changes in the chemical exchange properties can be excluded. With this knowledge at hand, the dualCEST approach is utilized to investigate alterations of the proteome in glioblastoma brain tumors. 
Application of dualCEST in vivo. For examinations in humans, the dualCEST pulse sequence was implemented on a clinically approved 3 T MR tomograph. Transfer of the dualCEST approach to lower $\mathrm{B}_{0}$, while maintaining its specificity to proteins, is enabled by the newly introduced dimension of selectivity. As stated above, this selectivity is generated by the coupling of two different CEST signals which makes the requirement of high spectral resolutions unnecessary. Moreover, the reduced spectral resolution at lower $\mathrm{B}_{0}$ is beneficial for dualCEST measurements leading to an increased signal strength (Supporting Information, Fig. S3). The reason for this is the increased number of proton species saturated at each frequency offset when the spectral resolution is reduced ${ }^{11,44}$. Consequently, the signal strength can further be enhanced by simply increasing the spectral bandwidth of the saturation pulses, i.e. reducing the pulse length $t_{p}$. Furthermore, the dualCEST signal strongly increases as a function of the pulse amplitude $B_{1}$ (Supporting Information, Fig. S3) offering an additional capability for signal amplification. Remarkably, although tuning of $t_{p}$ and $B_{1}$ leads to extensive peak broadening, dualCEST again maintains its specificity. In this study, $t_{p}=20 \mathrm{~ms}$ and $B_{1}=$ $2 \mu \mathrm{T}$ were found to be optimal at $\mathrm{B}_{0}=3 \mathrm{~T}$ in terms of maximizing the signal strength while avoiding direct water saturation. In addition, the dualCEST signal is approximately linear in $\mathrm{B}_{1}$ at the specified parameters allowing simple correction for $\mathrm{B}_{1}$-inhomogeneities in living tissue. In the measurement of a glioblastoma patient, the isolated protein signal $\mathrm{T}_{\text {protein }}$ is significantly

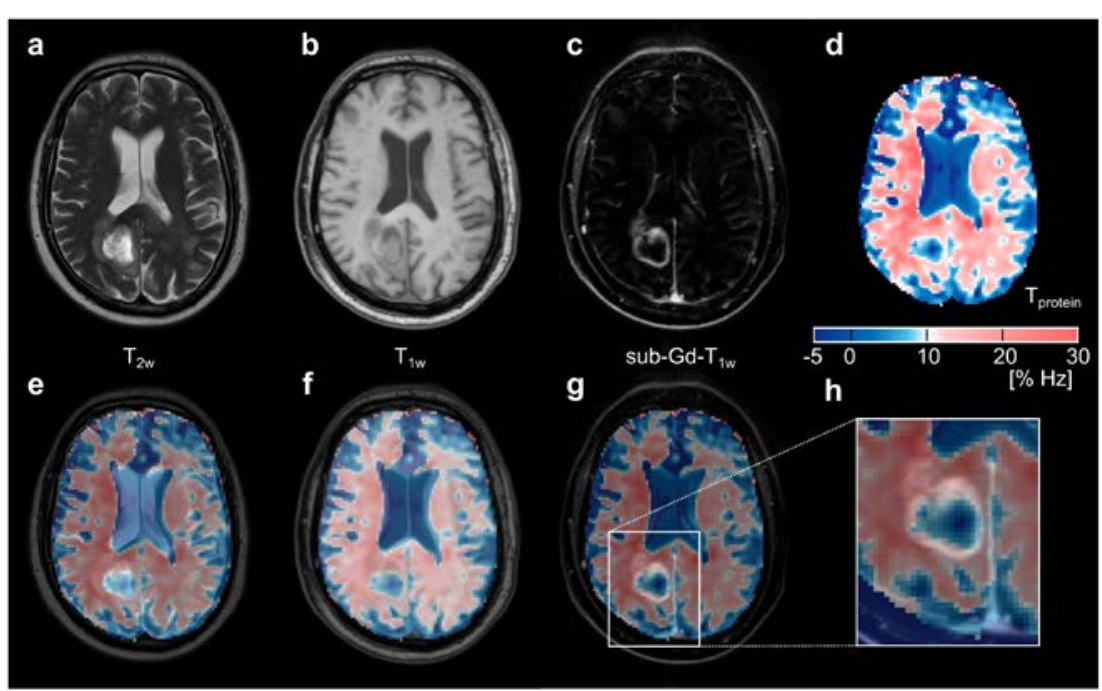

Figure 6 | in vivo dualCEST examination. Multi-parametric MR images of a glioblastoma brain tumor patient. (a-c) Conventional MR contrasts: $T_{2 w^{-}}, T_{1 w^{-}}$weighted and subtracted gadolinium contrast enhanced $\mathrm{T}_{1 w}$-weighted MRI. (d) The unbiased dualCEST signal of proteins. (e-h) Fusion of conventional MR contrasts and the dualCEST image.

reduced in the necrotic tumor region in comparison to normal appearing white matter (Fig. 6h). Edges are clearly defined and show a precise correlation with the inner part of the ringenhancement in the contrast media enhanced image. The signal strength $T_{\text {protein }}$ in necrotic tissue is comparable to values in the cerebrospinal fluid (CSF) suggesting a considerable degeneration of the proteome in the tumor core. The exceptional specificity to proteins allows now to unambiguously assign the observed signal drop to a reduced protein content or denaturation of the global protein folding state. Functioning of the dualCEST measurement is confirmed by signal values around zero in the CSF, in which a negligible concentration of proteins can be assumed. The slight mismatch of the dualCEST image with the anatomical structure of the ventricular system might be caused by head movements during the examination. In contrast, anatomical structures between brain matter and peripheral CSF show an excellent correlation with $T_{\text {protein. }}$ In a region of interest analysis, dualCEST signal values in gray and white matter brain regions are not distinguishable. All in all, the presented in vivo image data verify the successful application of dualCEST examinations in humans enabling further investigations of the proteome in several pathologies. 


\section{Discussion}

The approach of dualCEST enables the unbiased detection of proteins in living organisms. On the contrary, in conventional Z-spectra in vivo the CEST signals of proteins are spectrally overlapping with signals originating from other cellular compounds (Fig. 1). In the past, several attempts have already been made to overcome this obstacle and to highlight the signal component of proteins. Ideas range from multi-parametric fitting ${ }^{14,15,45-47}$, over several types of exchange rate filters $33,34,48,35,49-51$, to suppression of confounding signals by a simultaneous pre-saturation at various frequency offsets ${ }^{52-55}$. Although these approaches work appropriately and allow to isolate e.g. the prominent amide proton resonance at $\Delta \omega=+3.5 \mathrm{ppm}$ or the rNOECEST signal of aliphatic protons around $\Delta \omega=-3.5 \mathrm{ppm}$, assignment of these signals to proteins remains to be questionable. With respect to the amide proton signal, a considerable part originates also from small peptides (i.e. di-peptides) and metabolites ${ }^{39}$. In addition, the aliphatic rNOE-CEST signal comprises contributions from other mobile macromolecules as e.g. lipids or saccharides ${ }^{26,14}$. In both cases, magnetization transfer rates to water are comparable to the mechanisms in proteins preventing a separation by exchange rate filtering. In this study, we introduce a novel dimension of selectivity which is based on the cross magnetization transfer between two particular CEST signals - termed as saturation crosstalk $\mathrm{T}_{\Delta \omega c}(\Delta \omega)$. Detection of the saturation crosstalk between amide and aliphatic protons allows a unique specificity to proteins, because only proteins exhibit individual CEST signals of both proton types. Specificity to proteins was verified experimentally by the successive addition of different cellular compounds to a protein model solution (Fig. 4). Moreover, we demonstrated that the dualCEST signal is intrinsically corrected for changes in water relaxation (i.e. $T_{1 w}$, Fig. 4d) as well as independent on chemical exchange properties (i.e. exchange rates, Fig. 5). This makes the dualCEST signal very robust against other physiological process, as e.g. $\mathrm{pH}$ changes, justifying the designation "unbiased". Parameters that in turn affect the amplitude of the dualCEST signal were identified to be the protein content as well as the protein folding state (Fig. 5) making dualCEST a valuable diagnostic tool to detect aberrant proteomes in vivo.

For the application in living organisms, the dualCEST approach offers some remarkable features to increase the signal-to-noise ratio (SNR). The largest drawback of the presented method is the intrinsically small SNR in comparison to conventional CEST acquisitions. The reason for this is the quite large number of nine Z-values required for the calculation of the isolated protein signal $\mathrm{T}_{\text {protein }}$ (equation (2)). Additionally, some of the Z-values appear more than once in the equation leading to noisy images. However, the newly introduced dimension of selectivity opens up new possibilities to overcome this obstacle. Because specificity of the dualCEST signal to proteins relies on the coupling of two signals rather than on resolving individual resonances, no high spectral resolutions are required. This enables application of high $B_{1}$ values to increase the signal strength without the loss of specificity (Supporting Information, Fig. S3). In contrast, spectral selectivity is clearly deteriorated when acquiring conventional Z-spectra at high $B_{1}$ due to extensive peak broadening. Another possibility to further amplify the strength of the dual CEST signal is to incorporate more protons at neighboring chemical shifts into the measurement procedure, i.e. saturating a defined spectral region instead of a single frequency offset. This considers the fact that in proteins the chemical shift of a single species of protons (e.g. amides) is dispersed depending on the location inside the protein and the respective chemical environment. Expansion of the spectral bandwidth of the pre-saturation pulses was realized by decreasing their length $t_{p}$. In this case, again the exceptional selectivity of the dualCEST approach allows to preserve the specificity to proteins although peaks are broadening. In addition, also the spectral resolution can be reduced to likewise saturate more protons and thus increase the signal strength. Consequently, the dualCEST signal profits from lower static magnetic field strengths, paving the way for dualCEST examinations on clinically approved MR tomographs. The increase in signal strength at lower $\mathrm{B}_{0}$ was verified experimentally (Supporting Information, Fig. S3). In this study, in vivo measurements were performed at a static magnetic field strength of $3 \mathrm{~T}$ and optimized to maximize the signal amplitude while avoiding direct water saturation. Normally, these features would allow the acquisition of dualCEST images with adequate SNR. However, in our 
specific case technical limitations of the utilized MR tomograph (please note: restricted amplifier performance but not specific absorption rate (SAR) limitations) prevented a presaturation with an ideal DC of $49 \%$. Instead, only an approximately halved DC could be realized which is why several repetitions were acquired to gain a reliable dualCEST image.

For repeated measurements, the dualCEST approach profits from a fast and direct acquisition. By "direct" we mean that the saturation crosstalk can be calculated from unprocessed data without the application of any fitting procedure to approximate e.g. the direct water saturation or SSMT. This allows a fast determination of the dualCEST signal without the need for sampling of an entire spectrum leading to a considerable saving in time. Exclusion of the fitting procedure was enabled by an analytical description based on the AREX ${ }^{40}$ evaluation (Supporting Information, Fig. S1). In this theory, $\mathrm{T}_{\Delta \omega c}(\Delta \omega)$ is a correction term - considering the exchange of magnetization between two different CEST pools - which is added to the longitudinal relaxation rate in the rotating frame $R_{1 \rho}$ of a multi pool system ${ }^{56-58}$. The saving in time was used to repeat the measurement and to accumulate sufficient SNR for dualCEST examinations in brain tumor patients.

The dualCEST approach allows to identify pathological modifications of the proteome in vivo. Significant changes of the isolated protein signal $T_{\text {protein }}$ were detected in the necrotic region of a human brain tumor (Fig. 6). Necrotic tissue is known to consist of decomposed dead cells verifying the ability of the dualCEST signal to detect aberrant proteomes in living organisms. In the future, whole collectives of patients have to be examined to reliably determine the actual contribution of proteins to CEST signal changes in different pathologies and to further investigate the diagnostic value of the presented method. Applications range from follow-up studies of cancer treatment, to the diagnosis of neurodegenerative diseases which are associated with the accumulation of pathogenic protein plaques, as e.g. Alzheimer's. Additionally, the approach of dualCEST should be particularly suitable to selectively detect binding mechanisms of proteins, since its specificity relies on the coupling of different CEST signals. Besides, selective imaging of small exogenous CEST agents binding to proteins is plausible, due to immobilization of the small molecules and the resulting inter-molecular magnetization transfer pathway. Concluding, in this study a novel dimension of selectivity for CEST experiments is introduced opening up a new branch in the research field of CEST with the opportunity for several new applications with an exceptional specificity. 


\section{Methods}

Model solutions. In total, 35 model solutions containing different amounts of BSA, SDS, creatine, carnosine, lipids and tissue homogenates were prepared. A detailed list of all experimental parameters is presented in the Supporting Information (Table S1). Unless otherwise specified, model solutions were buffered at $\mathrm{pH} 7$ using phosphate-buffered saline (PBS). Final $\mathrm{pH}$ was checked by means of a calibrated $\mathrm{pH}$-electrode and discrepancies (e.g. in the case of high solute concentrations) were corrected using $\mathrm{NaOH}$ or $\mathrm{HCl}$. All model solutions were pipetted from high-concentrated stock solutions to ensure precise adjustment of different concentrations. Samples containing ex vivo tissue components were continuously chilled on ice before measurement. Protein-free brain lipids were extracted from mouse brain tissue with tetrahydrofuran, filtered and lyophilized for liposome preparation (size $120 \mathrm{~nm}$ ) throughout sonication. Tissue homogenates were made from white matter pig brain tissue and prepared according to previous studies ${ }^{44}$.

Subjects. One patient with a newly diagnosed and histologically proven glioblastoma was examined before surgery. Approval of the local ethics committee after written informed consent was obtained.

CEST spectroscopy. Model solutions were examined on a $14.1 \mathrm{~T}\left(600.13 \mathrm{MHz}\right.$ for $\left.{ }^{1} \mathrm{H}\right)$ narrow bore Avance II spectrometer (Bruker BioSpin, Karlsruhe-Rheinstetten, Germany). A 5-mm or 8-mm probe was used for RF irradiation and signal acquisition. To avoid radiation damping the probes were operated in detuned mode. Temperature of the samples was stabilized at 25 or $37^{\circ} \mathrm{C}$ using the internal heating and cooling device. For conventional CEST measurements, pre-saturation at $\Delta \omega$ was achieved by Gaussian-shaped RF pulses of mean amplitude $B_{1}=$ flip angle $/\left(\gamma \cdot t_{p}\right)$, length $t_{p}$ and duty cycle $D C=t_{p} /\left(t_{p}+t_{d}\right)$. In the case of dualCEST, an additional Gaussian-shaped RF pulse $\left(\Delta \omega_{c}\right)$ of same amplitude and length was centered in the middle of the interpulse delay $t_{d}$ (Fig. 2a). A detailed list of all experimental parameters is presented in the Supporting Information (Table S1). The overall duration of the pre-saturation period complied with the criterion for steady-state measurements: $t_{s a t} » T_{1 w}$. Z-values were calculated by integration of the water resonance in the range of $\pm 0.45 \mathrm{ppm}\left(\mathrm{M}_{\mathrm{sat}}\right)$ and normalized with the equilibrium magnetization $\left(\mathrm{M}_{0}\right): \mathrm{Z}=\mathrm{M}_{\mathrm{sat}} / \mathrm{M}_{0}$. To compensate for systematic signal fluctuations, $M_{0}$ was acquired at different time points and interpolated to obtain an individual $M_{0}$ for each pre-saturation cycle. Conventional Z-spectra were sampled at 118 frequency offsets in unequal steps between $\pm 150 \mathrm{ppm}$. Isolated CEST signals, compensated for direct water saturation, SSMT and water relaxation properties were calculated at each frequency offset $\Delta \omega$ using the AREX ${ }^{40}$ evaluation: $\operatorname{AREX}(\Delta \omega)=\frac{R_{1 w}}{D C} \cdot\left(\frac{1}{z(\Delta \omega)}-\frac{1}{z_{\text {ref }}(\Delta \omega)}\right)$. $R_{1 w}$ was measured with a saturationrecovery sequence. The reference spectrum $Z_{\text {ref }}$ was estimated by a multi-parametric fit: $Z_{\text {ref }}=1-\sum_{i} L_{i}$, where $L_{i}$ are Lorentzian-shaped functions representing the direct water saturation and SSMT. To avoid contributions from CEST signals, as well as to take into account the broadening of the direct water saturation due to a pulsed pre-saturation, data points in the range from \pm 10 to $\pm 0.5 \mathrm{ppm}$ and in between $\pm 0.2 \mathrm{ppm}$ were excluded from the fitting procedure. Displayed errors were calculated by the standard deviation of repeated measurements.

dualCEST MRI. In vivo measurements were performed on a $3 \mathrm{~T}\left(123.26 \mathrm{MHz}\right.$ for $\left.{ }^{1} \mathrm{H}\right)$ wholebody MR-PET tomograph (Biograph mMR; Siemens Healthcare $\mathrm{GmbH}$, Erlangen, Germany) using a custom developed CEST pulse sequence, based on a two-dimensional Half Fourier Acquisition Single Shot Turbo Spin Echo (HASTE) readout, and a 16-channel Siemens mMR Head/Neck A Tim Coil. Msat images (matrix $128 \times 102$, resolution $1.88 \times 1.88 \times 5 \mathrm{~mm}^{3}$ ) were acquired after pre-saturation with in total 167 Gaussian-shaped RF pulses of $B_{1}=2 \mu T, t_{p}=20$ $\mathrm{ms}$ and $\mathrm{DC}=28 \%$ leading to $\mathrm{t}_{\mathrm{sat}}=6 \mathrm{~s}$. Due to peak broadening of the dualCEST signal using the specified saturation parameters, $\Delta \omega_{c}$ in equation (2) was adjusted to -5 and $-30 \mathrm{ppm}$ for protein and SSMT signals, respectively. All images were co-registered to avoid motion artefacts. According to CEST spectroscopy, several $\mathrm{M}_{0}$ images were acquired at different time points and interpolated to yield an individual $M_{0}$ for each Z-image. To increase SNR, Z-images 
were averaged by 18 acquisitions and smoothed by a Gaussian-kernel ( $\sigma=1$ pixel). The final dualCEST contrast (equation (2)) was corrected for $B_{1}$-inhomogeneities by means of the onepoint "contrast-correction" method as described in a previous study ${ }^{59}$. $\mathrm{B}_{1}$ was determined by the simultaneous mapping of the water shift and $\mathrm{B}_{1}$ (WASABI ${ }^{60}$ approach using the same CEST pulse sequence with adjusted pre-saturation parameters. $T_{1 w}$ mapping was achieved by fitting $T_{1 w}$-weighted images of a saturation recovery HASTE sequence. In total, the overall measurement time was approximately 22 min, comprising 18 min dualCEST, 2 min WASABI and 2 min $\mathrm{T}_{1 \mathrm{w}}$ mapping. 


\section{References}

1. Stryer, L. Biochemie. (Spektrum Akademischer Verlag, 1996).

2. Taylor, R. C. \& Dillin, A. Aging as an Event of Proteostasis Collapse. Cold Spring Harb. Perspect. Biol. 3, a004440 (2011).

3. Hanahan, D. \& Weinberg, R. A. The Hallmarks of Cancer. Cell 100, 57-70 (2000).

4. Hanahan, D. \& Weinberg, R. A. Hallmarks of Cancer: The Next Generation. Cell 144, 646674 (2011).

5. Jucker, M. \& Walker, L. C. Self-propagation of pathogenic protein aggregates in neurodegenerative diseases. Nature 501, 45-51 (2013).

6. Wanker, E. E. Protein Aggregation and Pathogenesis of Huntington's Disease: Mechanisms and Correlations. Biol. Chem. 381, 937-942 (2005).

7. Wolff, S. D. \& Balaban, R. S. NMR imaging of labile proton exchange. J. Magn. Reson. 1969 86, 164-169 (1990).

8. Ward, K. m. \& Balaban, R. s. Determination of $\mathrm{pH}$ using water protons and chemical exchange dependent saturation transfer (CEST). Magn. Reson. Med. 44, 799-802 (2000).

9. Zhou, J. \& Zijl, P. C. M. van. Chemical exchange saturation transfer imaging and spectroscopy. Prog. Nucl. Magn. Reson. Spectrosc. 48, 109-136 (2006).

10. McMahon, M. T., Gilad, A. A., Bulte, J. W. M. \& Zijl, P. C. M. V. Chemical Exchange Saturation Transfer Imaging: Advances and Applications. (Pan Stanford Publishing Pte Ltd, 2017).

11. van Zijl, P. C. M., Lam, W. W., Xu, J., Knutsson, L. \& Stanisz, G. J. Magnetization Transfer Contrast and Chemical Exchange Saturation Transfer MRI. Features and analysis of the field-dependent saturation spectrum. Neurolmage Published Online (2017). doi:10.1016/j.neuroimage.2017.04.045

12. Zhou, J., Payen, J.-F., Wilson, D. A., Traystman, R. J. \& van Zijl, P. C. M. Using the amide proton signals of intracellular proteins and peptides to detect $\mathrm{pH}$ effects in MRI. Nat. Med. 9, 1085-1090 (2003). 
13. Jones, C. K. et al. Amide proton transfer imaging of human brain tumors at 3T. Magn. Reson. Med. 56, 585-592 (2006).

14. Jones, C. K. et al. Nuclear Overhauser enhancement (NOE) imaging in the human brain at 7 T. Neurolmage 77, 114-124 (2013).

15. Zaiss, M. et al. Relaxation-compensated CEST-MRI of the human brain at $7 \mathrm{~T}$ : Unbiased insight into NOE and amide signal changes in human glioblastoma. Neurolmage 112, 180-188 (2015).

16. Heo, H.-Y. et al. Whole-brain amide proton transfer (APT) and nuclear overhauser enhancement (NOE) imaging in glioma patients using low-power steady-state pulsed chemical exchange saturation transfer (CEST) imaging at 7T. J. Magn. Reson. Imaging 44, $41-50$ (2016).

17. Zhou, J. et al. Differentiation between glioma and radiation necrosis using molecular magnetic resonance imaging of endogenous proteins and peptides. Nat. Med. 17, 130-134 (2011).

18. Mehrabian, H., Desmond, K. L., Soliman, H., Sahgal, A. \& Stanisz, G. J. Differentiation between Radiation Necrosis and Tumor Progression Using Chemical Exchange Saturation Transfer. Clin. Cancer Res. Published Online (2017). doi:10.1158/1078-0432.CCR-16-2265

19. Sun, P. Z., Zhou, J., Sun, W., Huang, J. \& Zijl, P. C. van. Detection of the Ischemic Penumbra Using pH-Weighted MRI. J. Cereb. Blood Flow Metab. 27, 1129-1136 (2007).

20. Sun, P. Z., Zhou, J., Huang, J. \& van Zijl, P. Simplified quantitative description of amide proton transfer (APT) imaging during acute ischemia. Magn. Reson. Med. 57, 405-410 (2007).

21. Cai, K. et al. Magnetic resonance imaging of glutamate. Nat. Med 18, 302-307 (2012).

22. Haris, M. et al. Exchange rates of creatine kinase metabolites: feasibility of imaging creatine by chemical exchange saturation transfer MRI. NMR Biomed. 25, 1305-1309 (2012). 
23. Rerich, E., Zaiss, M., Korzowski, A., Ladd, M. E. \& Bachert, P. Relaxation-compensated CEST-MRI at $7 \mathrm{~T}$ for mapping of creatine content and $\mathrm{pH}$ - preliminary application in human muscle tissue in vivo. NMR Biomed. 28, 1402-1412 (2015).

24. Walker-Samuel, S. et al. In vivo imaging of glucose uptake and metabolism in tumors. Nat. Med. 19, 1067-1072 (2013).

25. Schuenke, P. et al. Fast and Quantitative T1p-weighted Dynamic Glucose Enhanced MRI. Sci. Rep. 7, 42093 (2017).

26. van Zijl, P. C. M. et al. Mechanism of Magnetization Transfer During On-Resonance Water Saturation. A New Approach to Detect Mobile Proteins, Peptides, and Lipids. Magn Reson Med 49, 440-449 (2003).

27. Henkelman, R. M., Stanisz, G. J. \& Graham, S. J. Magnetization transfer in MRI: a review. NMR Biomed. 14, 57-64 (2001).

28. Henkelman, R. M. et al. Quantitative interpretation of magnetization transfer. Magn. Reson. Med. 29, 759-766 (1993).

29. Zaiss, M. et al. A combined analytical solution for chemical exchange saturation transfer and semi-solid magnetization transfer. NMR Biomed. 28, 217-230 (2015).

30. Goerke, S., Zaiss, M. \& Bachert, P. Characterization of creatine guanidinium proton exchange by water-exchange (WEX) spectroscopy for absolute-pH CEST imaging in vitro. NMR Biomed. 27, 507-518 (2014).

31. Liepinsh, E. \& Otting, G. Proton exchange rates from amino acid side chainsimplications for image contrast. Magn. Reson. Med. 35, 30-42 (1996).

32. Bai, Y., Milne, J. S., Mayne, L. \& Englander, S. W. Primary structure effects on peptide group hydrogen exchange. Proteins Struct Funct Bioinf 17, 75-86 (1993).

33. Friedman, J. I., McMahon, M. T., Stivers, J. T. \& Van Zijl, P. C. M. Indirect Detection of Labile Solute Proton Spectra via the Water Signal Using Frequency-Labeled Exchange (FLEX) Transfer. J. Am. Chem. Soc. 132, 1813-1815 (2010).

34. Zu, Z. et al. A new method for detecting exchanging amide protons using chemical exchange rotation transfer. Magn. Reson. Med. 69, 637-647 (2013). 
35. Xu, J. et al. Variable Delay Multi-Pulse Train for Fast Chemical Exchange Saturation Transfer and Relayed-Nuclear Overhauser Enhancement MRI. Magn. Reson. Med. 71, 1798-1812 (2014).

36. Zaiss, M., Kunz, P., Goerke, S., Radbruch, A. \& Bachert, P. MR imaging of protein folding in vitro employing Nuclear-Overhauser-mediated saturation transfer. NMR Biomed. 26, 1815-1822 (2013).

37. Longo, D. L. et al. Chemical exchange saturation transfer (CEST): an efficient tool for detecting molecular information on proteins' behaviour. Analyst 139, 2687-2690 (2014).

38. Goerke, S. et al. Signature of protein unfolding in chemical exchange saturation transfer imaging. NMR Biomed. 28, 906-913 (2015).

39. Goerke, S. et al. Aggregation-induced changes in the chemical exchange saturation transfer (CEST) signals of proteins. NMR Biomed. 30, e3665 (2017).

40. Zaiss, M. et al. Inverse Z-spectrum analysis for spillover-, MT-, and T1-corrected steady-state pulsed CEST-MRI - application to $\mathrm{pH}$-weighted MRI of acute stroke. NMR Biomed. 27, 240-252 (2014).

41. Neuhaus, D. \& Williamson, M. P. The Nuclear Overhauser Effect in Structural and Conformational Analysis. (Wiley, 1989).

42. van Zijl, P. C. M. \& Yadav, N. N. Chemical exchange saturation transfer (CEST): What is in a name and what isn't? Magn. Reson. Med. 65, 927-948 (2011).

43. Lin, V. J. C. \& Koenig, J. L. Raman studies of bovine serum albumin. Biopolymers 15, 203-218 (1976).

44. Zaiss, M. et al. Downfield-NOE-suppressed amide-CEST-MRI at 7 Tesla provides a unique contrast in human glioblastoma. Magn. Reson. Med. 77, 196-208 (2017).

45. Desmond, K. L., Moosvi, F. \& Stanisz, G. J. Mapping of Amide, Amine, and Aliphatic Peaks in the CEST Spectra of Murine Xenografts at 7 T. Magn. Reson. Med. 71, 18411853 (2013).

46. Heo, H.-Y., Zhang, Y., Lee, D.-H., Hong, X. \& Zhou, J. Quantitative assessment of amide proton transfer (APT) and nuclear overhauser enhancement (NOE) imaging with 
extrapolated semi-solid magnetization transfer reference (EMR) signals: Application to a rat glioma model at 4.7 tesla. Magn. Reson. Med. 75, 137-149 (2016).

47. Zhou, I. Y. et al. Quantitative chemical exchange saturation transfer (CEST) MRI of glioma using Image Downsampling Expedited Adaptive Least-squares (IDEAL) fitting. Sci. Rep. 7, 84 (2017).

48. Zu, Z. et al. Multi-angle ratiometric approach to measure chemical exchange in amide proton transfer imaging. Magn. Reson. Med. 68, 711-719 (2012).

49. $\mathrm{Xu}, \mathrm{X}$. et al. Magnetization transfer contrast-suppressed imaging of amide proton transfer and relayed nuclear overhauser enhancement chemical exchange saturation transfer effects in the human brain at 7T. Magn. Reson. Med. 75, 88-96 (2016).

50. $\mathrm{Xu}, \mathrm{J}$. et al. On-resonance variable delay multipulse scheme for imaging of fastexchanging protons and semisolid macromolecules. Magn. Reson. Med. 77, 730-739 (2017).

51. Lin, C.-Y. et al. Using frequency-labeled exchange transfer to separate out conventional magnetization transfer effects from exchange transfer effects when detecting ParaCEST agents. Magn. Reson. Med. 67, 906-911 (2012).

52. Scheidegger, R., Vinogradov, E. \& Alsop, D. C. Amide proton transfer imaging with improved robustness to magnetic field inhomogeneity and magnetization transfer asymmetry using saturation with frequency alternating RF irradiation. Magn. Reson. Med. 66, 1275-1285 (2011).

53. Lee, J.-S., Regatte, R. R. \& Jerschow, A. Isolating chemical exchange saturation transfer contrast from magnetization transfer asymmetry under two-frequency rf irradiation. J. Magn. Reson. 215, 56-63 (2012).

54. Friedman, J. I., Xia, D., Regatte, R. R. \& Jerschow, A. Transfer Rate Edited experiment for the selective detection of Chemical Exchange via Saturation Transfer (TRE-CEST). J. Magn. Reson. 256, 43-51 (2015).

55. Närväinen, J., Hubbard, P. L., Kauppinen, R. A. \& Morris, G. A. Z-spectroscopy with Alternating-Phase Irradiation. J. Magn. Reson. 207, 242-250 (2010). 
56. Zaiss, M. \& Bachert, P. Exchange-dependent relaxation in the rotating frame for slow and intermediate exchange - modeling off-resonant spin-lock and chemical exchange saturation transfer. NMR Biomed 26, 507-518 (2013).

57. Zaiss, M. \& Bachert, P. Chemical exchange saturation transfer (CEST) and MR Zspectroscopy in vivo: a review of theoretical approaches and methods. Phys. Med. Biol. 58, R221-R269 (2013).

58. Trott, O. \& Palmer III, A. G. Theoretical study of R1 $\rho$ rotating-frame and R2 freeprecession relaxation in the presence of n-site chemical exchange. J. Magn. Reson. 170, 104-112 (2004).

59. Windschuh, J. et al. Correction of B1-inhomogeneities for relaxation-compensated CEST imaging at 7 T. NMR Biomed. 28, 529-537 (2015).

60. Schuenke, P. et al. Simultaneous mapping of water shift and B1(WASABI)_Application to field-Inhomogeneity correction of CESTMRI data. Magn. Reson. Med. 77, 571-580 (2017). 


\section{Supporting Information (SI)}

\section{In vivo protein imaging by means of dual frequency irradiation chemical exchange saturation transfer (dualCEST)}

Steffen Goerke*, Johannes Breitling*, Moritz Zaiss, Johannes Windschuh, Patrick Schuenke, Daniel Paech, Dario Livio Longo, Karel D. Klika, Mark E. Ladd, and Peter Bachert

\section{Mathematical derivation of the dualCEST formula (equation (1))}

As demonstrated in the manuscript (Fig. 2c), the saturation crosstalk $\mathrm{T}_{\Delta \omega c}(\Delta \omega)$ can be quantified by subtraction of the two AREX spectra with and without constant saturation at $\Delta \omega_{c}$ :

$$
\mathrm{T}_{\Delta \omega_{C}}(\Delta \omega)=\operatorname{AREX}_{l}(\Delta \omega)-\operatorname{AREX}_{I I}(\Delta \omega)
$$

with the AREX spectra defined in Fig. S1.

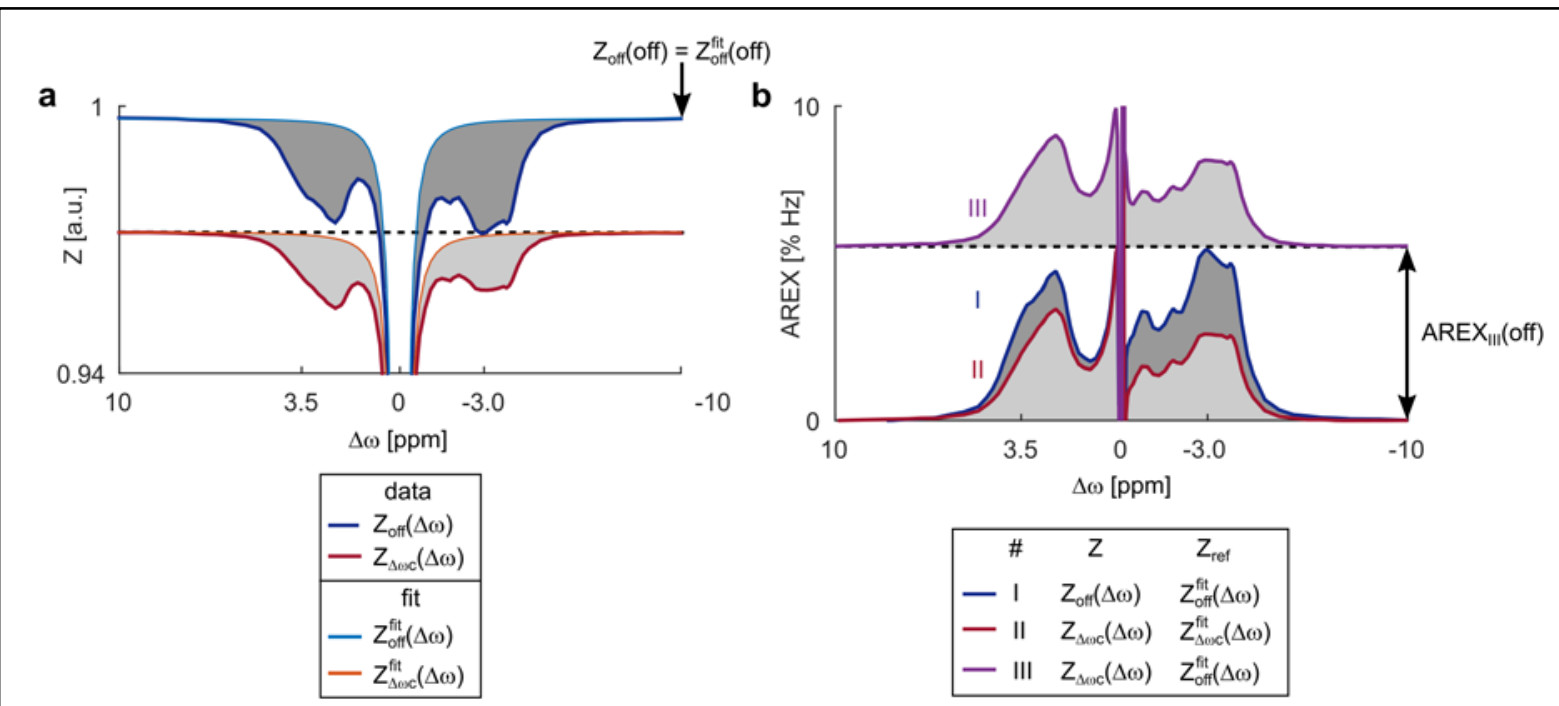

Figure S1 | The dualCEST formula. (a) Z-spectra of BSA proteins $\left(B_{1}=0.45 \mu T\right)$ with and without constant saturation at $\Delta \omega_{c}=-3.0 \mathrm{ppm}$. The direct water saturation was estimated by a Lorentzian fit. (b) AREX-spectra calculated by different combinations of $Z$ and $Z_{\text {ref. }}$.

As can be seen from the definitions, calculation of the two AREX spectra requires a fitting of the direct water saturation. To overcome this obstacle and to exclude the values which are determined by the fitting procedure $\left(\mathrm{Z}^{\mathrm{fit}}\right)$ the following relation is used:

$$
\operatorname{AREX}_{I I}(\Delta \omega)=\operatorname{AREX}_{I I I}(\Delta \omega)-\operatorname{AREX}_{\text {III }}(\text { off })
$$

Simple subtraction of the signal offset AREX at $\Delta \omega_{c}$ is possible due to the linearity of CEST signals using the AREX ${ }^{40}$ evaluation. A graphical illustration of relation (S2) for the case of $\Delta \omega_{c}=-3.0 \mathrm{ppm}$ is presented in Fig. S1b. Insertion of equation (S2) into (S1) together with the mathematical expression for AREX leads to:

$$
\mathrm{T}_{\Delta \omega_{\mathrm{C}}}(\Delta \omega)=\frac{\mathrm{R}_{1 \mathrm{w}}}{\mathrm{DC}} \cdot\left[\frac{1}{\mathrm{Z}_{\mathrm{off}}(\Delta \omega)}+\frac{1}{\mathrm{Z}_{\Delta \omega_{\mathrm{C}}}(\mathrm{off})}-\frac{1}{\mathrm{Z}_{\Delta \omega_{\mathrm{C}}}(\Delta \omega)}-\frac{1}{\mathrm{Z}_{\text {off }}^{\text {fiff }}}\right]
$$


Finally, the relation $Z_{\text {off }}($ off $)=Z_{\text {off }}^{\text {fit }}$ (off) (Fig. S1a) is used to completely bypass the fitting procedure and to yield equation (1).

\section{Mathematical background of the ssMT correction (equation (2))}

The presented method to correct for dualCEST signals of semi-solid macromolecular structures comprises two parts (Fig. 3): (i) sampling of the underlying SSMT component with a constant saturation off-resonant from protein signals $T_{-10}(\Delta \omega)$, and (ii) rescaling of this signal to the actual amplitude by the factor $\mathrm{T}_{-3.5}(+10) / \mathrm{T}_{-10}(+10)$. This approach can be explained from a mathematical point of view under the assumption that the dependence of $T$ on the frequency offsets $\Delta \omega$ and $\Delta \omega_{c}$ can be expressed as the product of two independent arbitrary functions:

$$
\mathrm{T}_{\Delta \omega_{\mathrm{C}}}(\Delta \omega)=\mathrm{t}_{\mathrm{C}}\left(\Delta \omega_{\mathrm{C}}\right) \cdot \mathrm{t}(\Delta \omega)
$$

In this case, the whole two dimensional space of $T$ spanned by $\Delta \omega$ and $\Delta \omega_{c}$ is completely defined by only two perpendicular lines $T_{\Delta \omega c}(+10)$ and $T_{-10}(\Delta \omega)$ (Fig. S2):

$$
T_{\Delta \omega_{C}}(\Delta \omega)=t_{C}\left(\Delta \omega_{C}\right) \cdot t(\Delta \omega)=\frac{t_{C}\left(\Delta \omega_{C}\right) t(+10)}{t_{C}(-10) t(+10)} \cdot t_{C}(-10) t(\Delta \omega)=\frac{T_{\Delta \omega_{C}}(+10)}{T_{-10}(+10)} \cdot T_{-10}(\Delta \omega)
$$

Consequently, the presented correction method enables the isolated determination of any superimposed signal $T$ by acquisition of three other frequency offset combinations (Fig. S2). In this study, the approach was used to quantify the signal component of semi-solid macromolecular structures $\mathrm{T}_{\text {sSMT }}$ contributing to the saturation crosstalk at $\Delta \omega_{c}=-3.5 \mathrm{ppm}$ (compare equation (2) and (S5)). Validity of assumption (S4) is justified by the demonstrated functionality of the presented correction method (Fig. 4).

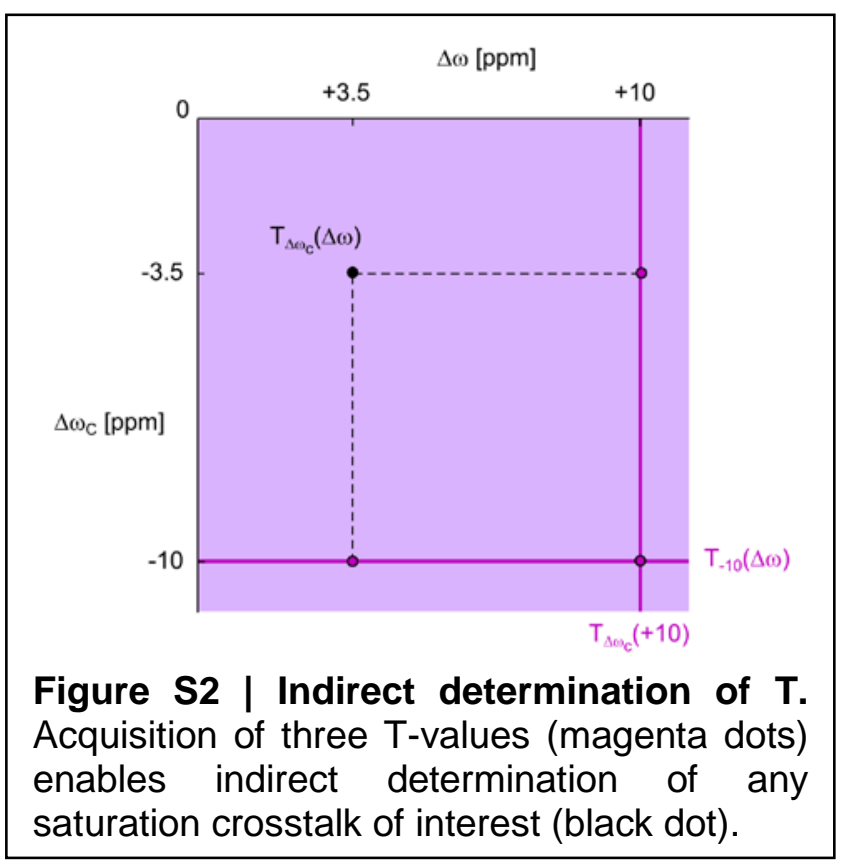

\section{Translation of dualCEST to clinical MR tomographs}

For in vivo examinations, the dualCEST pulse sequence parameters were optimized to maximize the signal strength $T$ while avoiding direct water saturation. Optimizations included parameters $B_{1}, t_{p}, t_{s a t}$ and $D C$ (data not shown). In addition, the influence of $B_{0}$ was investigated. For this purpose, the identical dualCEST pulse sequence was implemented on four different MR tomographs/ spectrometers. Remarkably, $T$ increases with decreasing $B_{0}$ 
(Fig. S3a) enabling translation of the dualCEST approach to clinically approved static magnetic field strengths $\left(B_{0} \leq 3 \mathrm{~T}\right)$. In doing so, the newly introduced dimension of selectivity allows to maintain the specificity to proteins although spectral resolution diminishes and peaks broaden.

To compensate for $\mathrm{B}_{1}$-inhomogeneities in living tissue the one-point "contrastcorrection" method ${ }^{59}$ was applied. This correction method assumes a linear relation of the image contrast as a function of $B_{1}$. Therefore, dualCEST images were acquired at $B_{1}=2 \mu T$ offering an adequate linear dependence of $T$ in the range of realistic $B_{1}$-variations of $\pm 20 \%$ in living tissue at $\mathrm{B}_{0}=3 \mathrm{~T}$ (Fig. S3b, gray shaded).

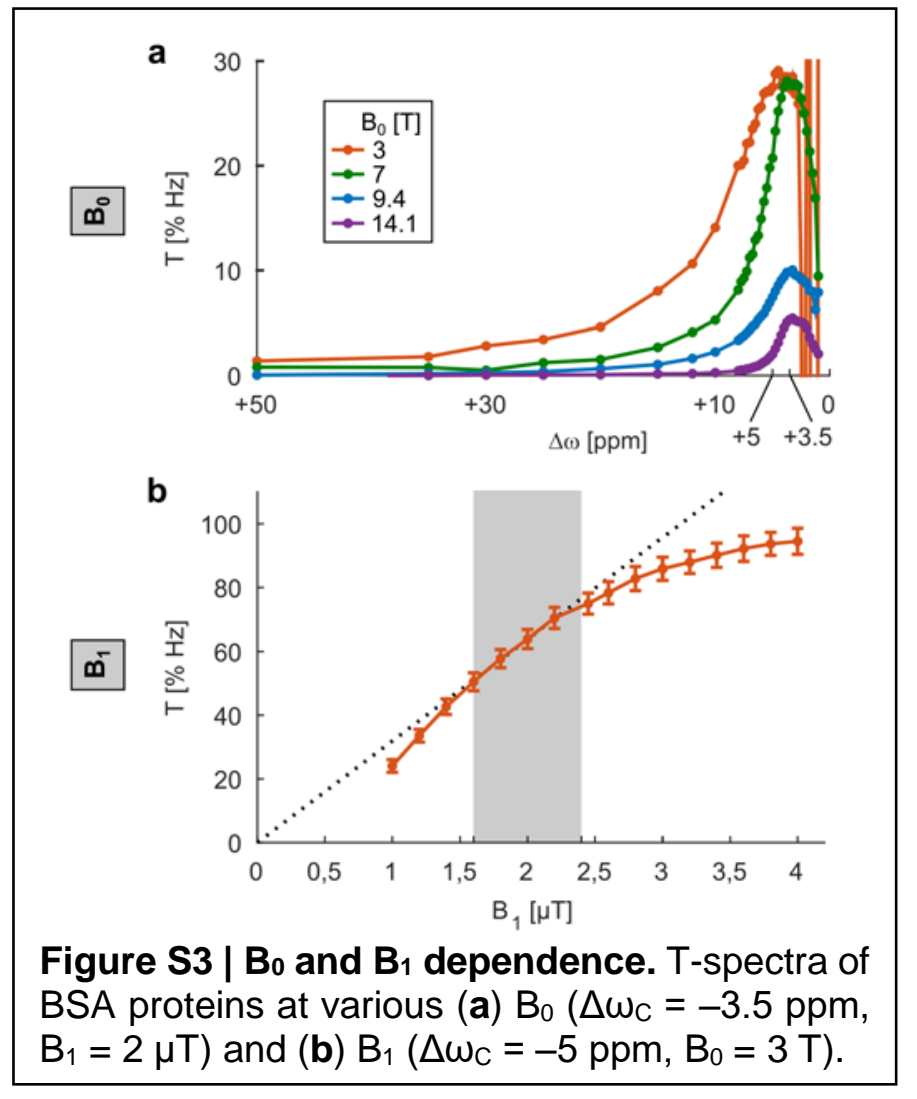




\section{Experimental Parameters}

In the following all compounds of the prepared model solutions, as well as the corresponding CEST pulse sequence parameters are listed and sorted by the illustrated figures.

\begin{tabular}{|c|c|c|c|c|c|c|c|c|c|c|c|}
\hline \multirow{2}{*}{ Fig. } & \multicolumn{4}{|c|}{ Model solution } & \multicolumn{7}{|c|}{ CEST } \\
\hline & compounds & $\mathrm{pH}$ & $\mathrm{T}\left[{ }^{\circ} \mathrm{C}\right]$ & $\mathrm{T}_{1 \mathrm{w}}[\mathrm{s}]$ & sequence & $B_{0}[T]$ & $B_{1}[\mu T]$ & $t_{\text {sat }}[s]$ & $t_{p}[\mathrm{~ms}]$ & DC [\%] & Rep. \\
\hline $\begin{array}{l}1, \\
3 a\end{array}$ & $66.7 \%(w / w)$ WM pig brain tissue & 7 & 37 & 2.45 & CEST & 14.1 & 0.75 & 12 & $\mathrm{cW}$ & 100 & 1 \\
\hline $\begin{array}{l}2, \\
\text { s1 }\end{array}$ & 10\% (w/v) BSA, $150 \mu \mathrm{M}$ Gd-DTPA & 7 & 25 & 0.97 & dualCEST & 14.1 & 0.45 & 5.5 & 100 & 49 & 1 \\
\hline $3 b$ & $66.7 \%(w / w)$ WM pig brain tissue & 7 & 25 & 2.23 & dualCEST & 14.1 & 1.5 & 12 & 100 & 49 & 1 \\
\hline \multirow{9}{*}{4} & $3 \%(w / v) B S A$ & \multirow{9}{*}{7} & \multirow{9}{*}{25} & 3.07 & \multirow{9}{*}{$\begin{array}{l}\text { dualCEST / } \\
\text { CEST }\end{array}$} & \multirow{9}{*}{14.1} & \multirow{9}{*}{$\begin{array}{l}1.5 / \\
1.5\end{array}$} & \multirow{9}{*}{$\begin{array}{l}12 / \\
20\end{array}$} & \multirow{9}{*}{$\begin{array}{l}7.8 / \\
100\end{array}$} & \multirow{9}{*}{$\begin{array}{l}49 / \\
49\end{array}$} & \multirow{9}{*}{$\begin{array}{c}10 / \\
1\end{array}$} \\
\hline & $3 \%(w / v) ~ B S A, 2.5 \mathrm{mM} \mathrm{Cr}, 25 \mathrm{mM}$ Car & & & 3.05 & & & & & & & \\
\hline & $3 \%(\mathrm{w} / \mathrm{v}) \mathrm{BSA}, 5 \mathrm{mM} \mathrm{Cr}, 50 \mathrm{mM}$ Car & & & 2.99 & & & & & & & \\
\hline & $3 \%(w / v)$ BSA, $7.5 \mathrm{mM} \mathrm{Cr}, 75 \mathrm{mM}$ Car & & & 2.94 & & & & & & & \\
\hline & $3 \%(\mathrm{w} / \mathrm{v}) \mathrm{BSA}, 10 \mathrm{mM} \mathrm{Cr}, 100 \mathrm{mM}$ Car & & & 2.89 & & & & & & & \\
\hline & $\begin{array}{c}3 \%(\mathrm{w} / \mathrm{v}) \mathrm{BSA}, 10 \mathrm{mM} \mathrm{Cr}, 100 \mathrm{mM} \text { Car, } \\
0.6 \%(\mathrm{w} / \mathrm{v}) \text { tissue-lipids }\end{array}$ & & & 2.83 & & & & & & & \\
\hline & $\begin{array}{c}3 \%(\mathrm{w} / \mathrm{v}) \mathrm{BSA}, 10 \mathrm{mM} \mathrm{Cr}, 100 \mathrm{mM} \text { Car, } \\
1.2 \%(\mathrm{w} / \mathrm{v}) \text { tissue-lipids }\end{array}$ & & & 2.78 & & & & & & & \\
\hline & $\begin{array}{c}3 \%(\mathrm{w} / \mathrm{v}) \mathrm{BSA}, 10 \mathrm{mM} \mathrm{Cr}, 100 \mathrm{mM} \text { Car, } \\
1.8 \%(\mathrm{w} / \mathrm{v}) \text { tissue-lipids }\end{array}$ & & & 2.73 & & & & & & & \\
\hline & $\begin{array}{c}3 \%(\mathrm{w} / \mathrm{v}) \mathrm{BSA}, 10 \mathrm{mM} \mathrm{Cr}, 100 \mathrm{mM} \text { Car, } \\
2.4 \%(\mathrm{w} / \mathrm{v}) \text { tissue-lipids }\end{array}$ & & & 2.69 & & & & & & & \\
\hline \multirow{6}{*}{$\begin{array}{l}5 a \\
5 b\end{array}$} & $3 \%(w / v) B S A$ & \multirow{6}{*}{7} & \multirow{6}{*}{25} & 3.08 & \multirow{6}{*}{ dualCEST } & \multirow{6}{*}{14.1} & \multirow{6}{*}{1.5} & \multirow{6}{*}{12} & \multirow{6}{*}{7.8} & \multirow{6}{*}{49} & \multirow{6}{*}{5} \\
\hline & $6 \%(w / v) B S A$ & & & 2.90 & & & & & & & \\
\hline & $9 \%(w / v) B S A$ & & & 2.74 & & & & & & & \\
\hline & $12 \%(w / v) B S A$ & & & 2.55 & & & & & & & \\
\hline & $15 \%(w / v) B S A$ & & & 2.39 & & & & & & & \\
\hline & $18 \%(w / v) B S A$ & & & 2.25 & & & & & & & \\
\hline & $2.5 \%(\mathrm{w} / \mathrm{v}) \mathrm{BSA}, 1.2 \mathrm{mM}$ SDS & & & 3.08 & & & & & & & \\
\hline & $2.5 \%(\mathrm{w} / \mathrm{v}) \mathrm{BSA}, 4.2 \mathrm{mM}$ SDS & & & 3.09 & & & & & & & \\
\hline & $2.5 \%(\mathrm{w} / \mathrm{v}) \mathrm{BSA}, 7.8 \mathrm{mM}$ SDS & & & 3.08 & & & & & & & \\
\hline & $2.5 \%(\mathrm{w} / \mathrm{v}) \mathrm{BSA}, 15 \mathrm{mM}$ SDS & & & 3.08 & & & & & & & \\
\hline $5 c$ & $2.5 \%(w / v) ~ B S A, 22.8 \mathrm{mM}$ SDS & & & 3.07 & & & & & & & \\
\hline $\mathbf{5 d}$ & $2.5 \%(\mathrm{w} / \mathrm{v}) \mathrm{BSA}, 30 \mathrm{mM}$ SDS & $r$ & 20 & 3.07 & Underter & 5.14 & 1.5 & 16 & 1.0 & 80 & 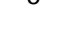 \\
\hline & $2.5 \%(w / v)$ BSA, $49.8 \mathrm{mM}$ SDS & & & 3.05 & & & & & & & \\
\hline & $2.5 \%(w / v) ~ B S A, 79.9 \mathrm{mM}$ SDS & & & 3.05 & & & & & & & \\
\hline & $2.5 \%(\mathrm{w} / \mathrm{v}) \mathrm{BSA}, 100.2 \mathrm{mM}$ SDS & & & 3.06 & & & & & & & \\
\hline & $2.5 \%(w / v) B S A, 139.9 \mathrm{mM}$ SDS & & & 3.04 & & & & & & & \\
\hline & & 6 & & 3.05 & & & & & & & \\
\hline & & 6.4 & & 3.04 & & & & & & & \\
\hline $\begin{array}{c}5 e, \\
5 f\end{array}$ & $3 \%(w / v) B S A$ & 7 & 25 & 3.08 & dualCEST & 14.1 & 1.5 & 12 & 7.8 & 49 & 5 \\
\hline & & 7.4 & & 3.03 & & & & & & & \\
\hline & & 7.8 & & 3.04 & & & & & & & \\
\hline & & & & 1.77 & & 3 & & 12 & & & 2 \\
\hline & & & & 2.03 & & 7 & & 12 & & & 2 \\
\hline & 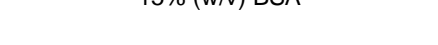 & $r$ & $2 u$ & 2.21 & Uudar LUT & 9.4 & 2 & 15 & 20 & & 1 \\
\hline & & & & 2.33 & & 14.1 & & 15 & & & 1 \\
\hline s3b & $20 \%(w / v) B S A$ & 7 & 25 & 1.08 & dualCEST & 3 & $1-4$ & 6 & 20 & 28 & 4 \\
\hline
\end{tabular}

Table S1 | Experimental parameters. Abbreviations: white matter (WM), continuous wave (cw), Magnevist ${ }^{\mathrm{TM}}$ Gadopentetic acid (Gd-DTPA), Creatine (Cr), Carnosine (Car) 